\title{
Heart rate variability in time and frequency domains: effects of gallopamil, nifedipine, and metoprolol compared with placebo
}

\begin{abstract}
Objective-To assess the effects of three different antianginal drugs on heart rate, blood pressure, and heart rate variability. Design-Randomised, single blind, placebo controlled, cross over study.

Setting-University hospital.

Participants-Nine healthy male volunteers.

Interventions-Oral administration of either $50 \mathrm{mg}$ gallopamil, $20 \mathrm{mg}$ nifedipine, $100 \mathrm{mg}$ metoprolol, or placebo according to a random crossover plan.

Main outcome measures-Time intervals between consecutive $R$ waves in electrocardiograms measured with an accuracy of $5 \mathrm{~ms}$ from digital Holter recordings. Blood pressure monitored continuously by finger plethysmography. Results-Metoprolol lowered heart rate from $62(6)$ to $51(5)$ beats $/ \mathrm{min}(p=0.003)$ after 78(23) minutes. Nifedipine provoked reflex tachycardia from $56(5)$ to 94(18) beats/min ( $<<0.001)$ at $10(3) \mathrm{min}$ utes after treatment followed by an exponential decline in heart rate to baseline values with a time constant of 34(7) min in seven subjects but 83 minutes in one volunteer. One subject showed no exponential decline in heart rate. Nifedipine significantly lowered the supine mean arterial pressure from $86(6)$ to $67(6) \mathrm{mm}$ Hg (p = 0.004) after 11(2) minutes, indicating an acute reduction in arterial resistance. Gallopamil did not significantly change mean heart rate or blood pressure. In the sitting position three hours after administration gallopamil and metoprolol significantly lowered power spectral density in the low frequency band $(0.03 \mathrm{~Hz}$ to $0.15 \mathrm{~Hz})$ compared with placebo $(p<0.05)$. Nifedipine did not produce such an effect.

Conclusions-Gallopamil and metoprolol both inhibit cardiac sympathetic activation compared with placebo, whereas nifedipine causes reflex sympathetic activation.

$R$ waves in an electrocardiogram ( $R R$ intervals) ${ }^{10-12}$ over several minutes. ${ }^{1314}$ The sympathetic cardiac innervation modifies the power spectral density mainly in a low frequency band (approximately from $0.03 \mathrm{~Hz}$ to 0.15 $\mathrm{Hz}$ ) and the parasympathetic innervation mainly in a higher frequency band (approximately from $0.15 \mathrm{~Hz}$ to $0.30 \mathrm{~Hz}) .^{213}$ In consequence, $\beta$ adrenergic blockers decrease low frequency power spectral density ${ }^{68}$ and atropine almost completely abolishes high frequency power spectral density. A change in posture from lying flat to sitting or standing and the reflex sympathetic activation increase low frequency power spectral density. In patients with autonomic neuropathy low and high frequency power spectral densities are suppressed. ${ }^{1516}$ Heart rate variability in the time domain ${ }^{17-19}$ is a marker of myocardial damage, ${ }^{20}$ is a reliable measure of cardiac parasympathetic activity, ${ }^{21}$ and, like baroreflex sensitivity, ${ }^{22}{ }^{23}$ may have prognostic implications $^{24} 25$ after myocardial infarction. ${ }^{26-29}$ In addition, the class 1c antiarrhythmic drugs flecainide and propafenone significantly decrease heart rate variability, ${ }^{30}$ which may be related to the effect of these drugs on mortality. ${ }^{31}$ So as far as spectral indices of cardiac autonomic innervation are concerned, however, there are only a few clinical reports on the pharmacodynamics of drugs with cardiovascular activity. Only one clinical study has suggested that after myocardial infarction $\beta$ adrenergic blockers and calcium channel blockers of the diltiazem type may decrease low frequency power spectral density, reflecting inhibition of cardiac sympathetic activation, whereas calcium channel blockers of the nifedipine type may not have this effect. ${ }^{32} \mathrm{We}$ assessed the effects on heart rate and blood pressure and on heart rate variability in the time and frequency domains of two different calcium channel blockers, gallopamil and nifedipine, to examine whether these calcium channel blockers inhibit cardiac sympathetic activation. We compared these data with those obtained with the $\beta$ adrenergic blocker metoprolol and with placebo.
\end{abstract}

(Br Heart f 1993;70:252-258)

Both sympathetic and parasympathetic cardiac tone can be assessed non-invasively ${ }^{1-3}$ and semiquantitatively ${ }^{4-6}$ by spectral analysis of heart rate variability. ${ }^{7-9}$ During sinus rhythm a spectral transformation is carried out on the time intervals between consecutive

\section{Subjects and methods}

The study was a randomised, placebo controlled, cross over trial and single blind for data evaluation. The experiments were carried out between July and October 1991. The study protocol was approved by the ethics committee of the Medical Faculty of the University of Heidelberg. 
Table 1 Individual characteristics of nine healthy male volunteers

\begin{tabular}{llll}
\hline Case no & Age (years) & Height $(\mathrm{cm})$ & Weight $(\mathrm{kg})$ \\
\hline 1 & 29 & 170 & 62 \\
2 & 26 & 172 & 63 \\
3 & 27 & 170 & 70 \\
4 & 29 & 185 & 72 \\
5 & 34 & 201 & 105 \\
6 & 29 & 175 & 67 \\
7 & 39 & 190 & 92 \\
8 & 27 & 172 & 74 \\
9 & 28 & 182 & 70 \\
Mean (SD) & $29 \cdot 8(4 \cdot 15)$ & $179 \cdot 7(10 \cdot 74)$ & $75 \cdot 0(14 \cdot 26)$ \\
\hline
\end{tabular}

\section{SUBJECTS}

Nine male volunteers participated in the study after they had given written informed consent. Table 1 shows their individual characteristics. The volunteers were healthy as judged by medical history, physical examination, electrocardiographic findings, and results of routine laboratory tests of blood and urine. All volunteers had normal sinus rhythm without extra beats, and they had no history of palpitations. None of the volunteers was a smoker, a heavily active sportsman, or taking drugs short term or long term.

\section{INTERVENTIONS}

The volunteers received orally either a tablet of placebo or $50 \mathrm{mg}$ gallopamil (Procorum, Minden Pharma), $100 \mathrm{mg}$ metoprolol (Lopresor, Ciba Geigy), or $20 \mathrm{mg}$ nifedipine (Adalat, Bayer). All drugs were given in immediate release formulations and administered according to a random cross over plan. There was a wash out phase of at least one week between the study days.

\section{EXPERIMENTAL PROCEDURES}

On the morning of each study day the volunteers were admitted to the research unit. A plastic cannula was inserted into a forearm vein as an emergency line. The digital Holterrecorder (Cardiolight, software revision 3.0; Medset, Hamburg) and a finger plethysmograph to measure blood pressure ${ }^{33}$ (2300 Finapres; Ohmeda, Madison, Wisconsin) were connected to the volunteer. The measurements started in all instances between $8 \mathrm{am}$ and $9 \cdot 15 \mathrm{am}$. For baseline data evaluation the volunteer rested supine for at least 15 minutes and then sat for another 15 minutes. Then the test drug was administered with $100 \mathrm{ml}$ of mineral water at room temperature, and the volunteer returned to supine position. Periods of 15 minutes' duration in the supine and sitting positions were repeated one hour and three hours after drug administration. Continuous Holter recordings and blood pressure measurements were maintained over the first four hours after drug administration. Two hours after receiving the drug the volunteer had a standardised breakfast.

Four hours after drug application a bicycle ergometry stress test was performed (Dynavit Conditronic S; Keiper, Rockenhausen, Germany). Initial performance was set to $50 \mathrm{~W}$ with increments of $25 \mathrm{~W}$ every two minutes. The exercise test was discontinued when the volunteer was exhausted. During exercise every minute an electrocardiogram of $15 \mathrm{sec}$ onds' duration was recorded from the chest leads V1 to V6. Every two minutes blood pressure was measured in the right arm by the Riva-Rocci method. During the next three hours the volunteer was allowed to walk around in the hospital under the surveillance of a physician. Thereafter he was discharged.

\section{DATA ACQUISITION AND PROCESSING}

The beat to beat values of heart rate were calculated from the corresponding RR intervals on the Holter electrocardiogram. The time resolution of the $R R$ interval measurements was $5 \mathrm{~ms}$. The beat to beat values of integrated mean arterial blood pressure were directly obtained from the plethysmograph and stored on an IBM compatible personal computer. Data processing was performed on this computer and the software was written in our laboratory in the programming languages Basic and C.

In the time domain the beat to beat values of heart rate and blood pressure were averaged every minute and plotted against the cumulative time. From these data the time between the baseline values before the administration of the test drug and the first peak or trough in heart rate and blood pressure after drug application were calculated. The changes in heart rate and blood pressure were obtained by subtracting the peak values from the baseline values. Heart rate variability in the time domain was assessed by counting the number of absolute changes greater than $50 \mathrm{~ms}$ between consecutive RR intervals in sinus rhythm during evaluation periods of 15 minutes' duration at baseline and one hour and three hours after drug administration.

In the frequency domain spectral analysis of $512 R R$ intervals was carried out from recordings during sinus rhythm according to the method of Rompelman et al. ${ }^{34}$ The RR intervals were first normalised by subtracting the mean $R R$ interval and dividing each $R R$ interval by the mean $R R$ interval. Then the Hanning function was applied to the data. The periodogram was calculated using the discrete fast Fourier transformation. The sampling frequency was calculated from the mean RR interval. Finally, to reduce the variance of the spectral estimates the power spectra were smoothed using an averaging technique. Every discrete spectral estimate was expressed as the mean of itself and its two neighbours. The power at the frequency zero and at the next two discrete frequencies was cut off (direct current removal) and total power spectral density was obtained by integration over the remaining values. As an index of cardiac sympathetic tone the low frequency power spectral density was obtained by integration of the power spectrum between $0.03 \mathrm{~Hz}$ and $0.15 \mathrm{~Hz}$. The high frequency power spectral density was calculated between $0 \cdot 15 \mathrm{~Hz}$ and $0.30 \mathrm{~Hz}$. Low and high frequency power spectral densities were 
expressed as percentages of total power spectral density.

\section{STATISTICAL TESTS}

Peak and trough values of heart rate and blood pressure in each volunteers were compared with baseline values with the KruskalWallis test. Power spectral densities of heart rate between different time phases within one study day were compared with the KruskalWallis test. Drug values were compared with placebo values with the paired $t$ test. The term significant means an error probability $p<0.05$ and the term highly significant denotes $p<0.001$. Data are expressed as means (SD).

\section{PHARMACODYNAMIC CALCULATIONS}

The exponential decline in heart rate after the rise of heart rate to peak values caused by nifedipine was examined by the following method. After the baseline heart rate before drug administration was subtracted from all values the data were transformed to the natural logarithm and a regression line was drawn through the values during the decline of heart rate. From the slope of this regression line the time constant of the exponential decline in heart rate $\left(t_{d}\right)$ was calculated. During $t_{d}$ heart rate (expressed as the average value every minute) declines to $1 / \mathrm{e}$ (with $\mathrm{e}$ as the basis of the natural logarithms, e = $2 \cdot 71828$ ) of its initial value.

\section{Results \\ HEART RATE AND TIME DOMAIN HEART RATE VARIABILITY}

Table 2 shows the numerical results for heart rate in the nine volunteers.

\section{Gallopamil}

When baseline values in each subject were compared gallopamil had no effect on heart rate. In case 2 , however, an atrioventricular block II type Mobitz occurred 40 minutes after drug application. It caused asymptomatic pauses with a duration of less than two seconds and lasted over 35 minutes. Heart rate while subjects were supine and sitting did not differ at baseline from values with placebo. After one hour supine heart rate was significantly higher (61 (5) beats/min $v 58$ (5) beats $/ \mathrm{min}$ ) and after three hours heart rate while sitting was significantly lower (72 (9) beats/min $v 76(10)$ beats/min). Heart rate variability did not significantly differ from values with placebo.

\section{Nifedipine}

All subjects showed a significant increase in heart rate while taking nifedipine. Two subjects (cases 1 and 5) had excessive reflex tachycardia with flushing and headache. The increase in heart rate was followed by an exponential decline to baseline values in almost all subjects. In one subject (case 2), however, the rapid increase in heart rate was followed by a rapid non-exponential decline to baseline value within four minutes. Heart rate was not significantly different at baseline from placebo values. After one hour heart rate had significantly increased in the supine position ( 68 (8) beats/min $v 58(5)$ beats $/ \mathrm{min}$ ) as well as in the sitting position (75 (7) beats $/ \min v 66(5)$ beats $/ \mathrm{min})$.

Heart rate variability significantly decreased (supine: 137 (130) v 295 (116); sitting: $87(80) v 162(80)$ ). After three hours the increase in heart rate was significant only in the supine position ( 69 (7) beats/min $v 66$ (7) beats/min) and heart rate variability did not differ from placebo values.

\section{Metoprolol}

Metoprolol significantly decreased heart rate compared to baseline values in all subjects from 62 (6) beats/min to 51 (5) beats/min within 78 (23) $\mathrm{min}$. Compared with placebo values heart rate and heart rate variability did not differ at baseline. Heart rate significantly decreased after one hour in the supine (54 (5) beats $/ \mathrm{min} v 58$ (5) beats $/ \mathrm{min}$ ) and sitting ( 57 (5) beats $/ \mathrm{min} v 66$ (5) beats/min) positions while heart rate variability significantly increased (supine: 371 (104) $v 295$ (116) sitting: 302 (101) $v 162$ (80). The effect on heart rate was still evident after three hours (supine: 61 (6) beats $/ \mathrm{min} v 66$ (7) beats $/ \mathrm{min}$; sitting: 63 (6) beats/min $v 76(10)$ beats $/ \mathrm{min}$ ). At this time heart rate variability significantly increased only in the sitting position (209 (111) $v 95$ (68)) compared with placebo.

Table 2 Heart rate variables after gallopamil, nifedipine, metoprolol, and placebo in nine healthy volunteers

\begin{tabular}{|c|c|c|c|c|c|c|c|c|c|c|c|c|c|c|c|c|c|}
\hline \multirow[b]{2}{*}{ Case } & \multicolumn{4}{|c|}{ Gallopamil } & \multicolumn{5}{|c|}{ Nifedipine } & \multicolumn{4}{|c|}{ Metoprolol } & \multicolumn{4}{|l|}{ Placebo } \\
\hline & $\begin{array}{l}\text { Baseline } \\
\text { (beats/ } \\
\text { no min) }\end{array}$ & $\begin{array}{l}\text { Peak or } \\
\text { trough } \\
\text { (beats/min) }\end{array}$ & $\begin{array}{l}\text { Time to } \\
\text { peak } \\
\text { (min) }\end{array}$ & $\begin{array}{l}R P P \\
(\text { mm } \\
\mathrm{Hg} / \mathrm{min})\end{array}$ & $\begin{array}{l}\text { Baseline } \\
\text { (beats/ } \\
\text { min) }\end{array}$ & $\begin{array}{l}\text { Peak or } \\
\text { trough } \\
\text { (beats/min) }\end{array}$ & $\begin{array}{l}\text { Time to } \\
\text { peak } \\
\text { (min) }\end{array}$ & $t_{d}(\min )$ & $\begin{array}{l}R P P \\
(\mathrm{~mm} \\
\mathrm{Hg} / \mathrm{min})\end{array}$ & $\begin{array}{l}\text { Baseline } \\
\text { (beats/ } \\
\text { min) }\end{array}$ & $\begin{array}{l}\text { Peak or } \\
\text { trough } \\
\text { (beats/min) }\end{array}$ & $\begin{array}{l}\text { Time to } \\
\text { peak } \\
\text { (min) }\end{array}$ & $\begin{array}{l}R P P \\
(\text { mm } \\
\mathrm{Hg} / \mathrm{min})\end{array}$ & $\begin{array}{l}\text { Baseline } \\
\text { (beats/ } \\
\text { min) }\end{array}$ & $\begin{array}{l}\text { Peak or } \\
\text { trough } \\
\text { (beats/min) }\end{array}$ & $\begin{array}{l}\text { Time to } \\
\text { peak } \\
\text { (min) }\end{array}$ & $\begin{array}{l}R P P \\
(\stackrel{m m}{H g / m i n})\end{array}$ \\
\hline $\begin{array}{l}1 \\
2 \\
3 \\
4 \\
5 \\
6 \\
7 \\
8 \\
9 \\
\text { Mean } \\
\text { (SD) }\end{array}$ & $\begin{array}{l}64 \\
66 \\
66 \\
56 \\
50 \\
50 \\
62 \\
65 \\
52 \\
59 \\
(7)\end{array}$ & $\begin{array}{l}58 \\
59 \\
63 \\
68 \\
57 \\
50 \\
62 \\
68 \\
52 \\
60 \\
(6)\end{array}$ & $\begin{array}{l}24 \\
24 \\
27 \\
66 \\
49 \\
\text { ND } \\
\text { ND } \\
30 \\
\text { ND } \\
37 \\
(17)\end{array}$ & $\begin{array}{l}22100 \\
23120 \\
27740 \\
31000 \\
28310 \\
28690 \\
22110 \\
24490 \\
30780 \\
26482 \\
(3575)\end{array}$ & $\begin{array}{l}65 \\
53 \\
58 \\
53 \\
49 \\
54 \\
63 \\
58 \\
55 \\
56 \\
(5)\end{array}$ & $\begin{array}{c}132 \\
74 \\
93 \\
83 \\
106 \\
75 \\
98 \\
98 \\
85 \\
94^{\star} \\
(18)\end{array}$ & $\begin{array}{l}7 \\
4 \\
11 \\
12 \\
13 \\
10 \\
9 \\
13 \\
13 \\
10 \\
(3)\end{array}$ & $\begin{array}{l}33 \\
\text { ND } \\
44 \\
83 \\
28 \\
32 \\
35 \\
42 \\
25 \\
34 \\
(7)\end{array}$ & $\begin{array}{l}22230 \\
20160 \\
26180 \\
19040 \\
24160 \\
26690 \\
15340 \\
22350 \\
37515 \\
23741 \ddagger \\
(6268)\end{array}$ & $\begin{array}{l}63 \\
68 \\
67 \\
60 \\
55 \\
57 \\
65 \\
70 \\
55 \\
62) \\
(6)\end{array}$ & $\begin{array}{l}57 \\
55 \\
50 \\
48 \\
47 \\
45 \\
52 \\
58 \\
47 \\
51 \dagger \\
(5)\end{array}$ & $\begin{array}{r}90 \\
90 \\
60 \\
60 \\
105 \\
60 \\
120 \\
60 \\
60 \\
78 \\
(23)\end{array}$ & $\begin{array}{l}18060 \\
14280 \\
13910 \\
17640 \\
22225 \\
19680 \\
13905 \\
22560 \\
24150 \\
18490 \ddagger \\
(3946)\end{array}$ & $\begin{array}{l}78 \\
58 \\
62 \\
61 \\
53 \\
50 \\
64 \\
63 \\
54 \\
60 \\
(8)\end{array}$ & $\begin{array}{l}78 \\
52 \\
55 \\
54 \\
60 \\
46 \\
64 \\
63 \\
51 \\
58 \\
(9)\end{array}$ & $\begin{array}{l}\text { ND } \\
30 \\
30 \\
30 \\
22 \\
30 \\
\text { ND } \\
\text { ND } \\
30 \\
29 \\
(3)\end{array}$ & $\begin{array}{l}21450 \\
20925 \\
37050 \\
30780 \\
29970 \\
28490 \\
20305 \\
24300 \\
35400 \\
27630 \\
(6256)\end{array}$ \\
\hline
\end{tabular}

${ }^{*} \mathrm{p}<0.001$ compared with baseline. $\mathrm{tp}=0.003$ compared with baseline. $\neq \mathrm{p}<0.05$ as compared with placebo. RPP $=$ rate-pressure product at maximal heart rate during exercise. $t_{d}=$ Time constant of exponential decline in heart rate. $\mathrm{ND}=$ not determinable. 
Table 3 Blood pressure variables after gallopamil, nifedipine, metoprolol, and placebo in nine healthy volunteers

\begin{tabular}{|c|c|c|c|c|c|c|c|c|c|c|c|c|}
\hline \multirow[b]{2}{*}{ Case no } & \multicolumn{3}{|l|}{ Gallopamil } & \multicolumn{3}{|l|}{ Nifedipine } & \multicolumn{3}{|l|}{ Metoprolol } & \multicolumn{3}{|l|}{ Placebo } \\
\hline & $\begin{array}{l}\text { Baseline } \\
(m m \mathrm{Hg})\end{array}$ & $\begin{array}{l}\text { Peak or trough } \\
(\mathrm{mm} \mathrm{Hg})\end{array}$ & $\begin{array}{l}\text { Time to } \\
\text { peak (min) }\end{array}$ & $\begin{array}{l}\text { Baseline } \\
(m m ~ H g)\end{array}$ & $\begin{array}{l}\text { Peak or trough } \\
(\mathrm{mm} \mathrm{Hg})\end{array}$ & $\begin{array}{l}\text { Time to } \\
\text { peak (min) }\end{array}$ & $\begin{array}{l}\text { Baseline } \\
(\mathrm{mm} \mathrm{Hg})\end{array}$ & $\begin{array}{l}\text { Peak or trough } \\
(\mathrm{mm} \mathrm{Hg})\end{array}$ & $\begin{array}{l}\text { Time to } \\
\text { peak (min) }\end{array}$ & $\begin{array}{l}\text { Baseline } \\
\text { (mm Hg) }\end{array}$ & $\begin{array}{l}\text { Peak or trough } \\
(\mathrm{mm} \mathrm{Hg})\end{array}$ & $\begin{array}{l}\text { Time to } \\
\text { peak (min) }\end{array}$ \\
\hline $\begin{array}{l}1 \\
2 \\
3 \\
4 \\
5 \\
6 \\
7 \\
8 \\
9 \\
\text { Mean (SD) }\end{array}$ & $\begin{array}{l}80 \\
82 \\
72 \\
78 \\
78 \\
77 \\
88 \\
82 \\
95 \\
81(7)\end{array}$ & $\begin{array}{l}88 \\
90 \\
93 \\
\text { ND } \\
78 \\
100 \\
95 \\
82 \\
95 \\
90(7)\end{array}$ & $\begin{array}{l}43 \\
47 \\
58 \\
\text { ND } \\
\text { ND } \\
47 \\
48 \\
\text { ND } \\
\text { ND } \\
49(6)\end{array}$ & $\begin{array}{c}88 \\
105 \\
95 \\
86 \\
80 \\
78 \\
90 \\
78 \\
95 \\
86(6)\end{array}$ & $\begin{array}{r}72 \\
157 \\
67 \\
70 \\
60 \\
97 \\
75 \\
61 \\
\text { ND } \\
67(6)\end{array}$ & $\begin{array}{r}12 \\
2 \\
14 \\
11 \\
8 \\
14 \\
14 \\
10 \\
\text { ND } \\
11(2)\end{array}$ & $\begin{array}{r}82 \\
82 \\
78 \\
80 \\
88 \\
70 \\
100 \\
80 \\
85 \\
83(8)\end{array}$ & $\begin{array}{l}98 \\
93 \\
80 \\
80 \\
70 \\
73 \\
100 \\
80 \\
85 \\
84(11)\end{array}$ & $\begin{array}{l}45 \\
50 \\
60 \\
\text { ND } \\
100 \\
80 \\
\text { ND } \\
\text { ND } \\
\text { ND } \\
67(23)\end{array}$ & $\begin{array}{l}75 \\
75 \\
93 \\
70 \\
83 \\
73 \\
80 \\
80 \\
85 \\
79(7)\end{array}$ & $\begin{array}{l}85 \\
75 \\
93 \\
87 \\
76 \\
73 \\
90 \\
80 \\
90 \\
83(7)\end{array}$ & $\begin{array}{l}30 \\
\text { ND } \\
\text { ND } \\
32 \\
42 \\
\text { ND } \\
40 \\
\text { ND } \\
32 \\
34(5)\end{array}$ \\
\hline
\end{tabular}

$\mathrm{ND}=$ not determinable.

Placebo

There were no effects on heart rate or heart rate variability.

\section{BLOOD PRESSURE}

Owing to a systematic effect of posture the integrated mean arterial blood pressure in the sitting position exceeded the pressure in the supine position by a mean of $25 \mathrm{~mm} \mathrm{Hg}$. Table 3 shows the numerical results for supine blood pressure only.

Gallopamil

Gallopamil showed no effect on blood pressure.

\section{Nifedipine}

Blood pressure decreased in six subjects from 86 (6) $\mathrm{mm} \cdot \mathrm{Hg}$ to 67 (6) $\mathrm{mm} \cdot \mathrm{Hg}$ within 11 (2) minutes after nifedipine administration. Two subjects (cases 2 and 6), however, showed an increase. Thirteen minutes after drug administration blood pressure measurement was discontinued in one subject (case 9) because of a technical problem until 30 minutes after drug administration. At this time the volunteer had the same blood pressure as at baseline.

\section{Metoprolol}

Compared with placebo metoprolol caused a slight but significant decrease in blood pressure three hours after administration in the supine position (placebo: 85 (10) $\mathrm{mm} \mathrm{Hg}$; metoprolol: 79 (11) $\mathrm{mm} \mathrm{Hg}$ ).

\section{Placebo}

Placebo had no effect on blood pressure.

RATE-PRESSURE PRODUCT WITH EXERCISE

Table 2 shows the numerical results. Although maximal workload did not differ between the drugs (in all subjects and under each drug it was 185 (23) Watts), nifedipine and metoprolol both significantly lowered maximal systolic blood pressure compared with placebo (nifedipine: 157 (24) $\mathrm{mm} \mathrm{Hg}$; metoprolol: 151 (17) $\mathrm{mm} \mathrm{Hg}$; placebo: 171 (24) $\mathrm{mm} \mathrm{Hg}$ ). In addition, metoprolol significantly lowered maximal heart rate under exercise (122 (15) beats $/ \mathrm{min}$ ) compared with placebo (160 (20) beats/min). With gallopamil maximal heart rate and systolic blood pressure during exercise did not differ from placebo values.

\section{SPECTRAL ANALYSIS OF HEART RATE}

\section{VARIABILITY}

Table 4 shows the low frequency power spectral density at different times after drug administration as an index of cardiac sympathetic tone. If there were high values of low frequency power spectral density $(>80)$ a single peak near $0.10 \mathrm{~Hz}$ was usually found. If there were low values of low frequency power spectral density $(<2)$ the main spectral power was usually found at frequencies lower than $0.03 \mathrm{~Hz}$. In $89 \%$ of all measurements the high power spectral density was below $10 \%$ of total power spectral density. Because of these irrelevant values and because there were no changes between the different phases or drugs the data are not presented in detail.

\section{Gallopamil}

Compared with placebo gallopamil significantly reduced the low frequency power spectral density after three hours in the sitting position. When compared with the preceding period in the supine position low frequency power spectral density did not increase in the sitting position at baseline and after one hour and three hours.

\section{Nifedipine}

Compared with placebo nifedipine significantly decreased low frequency power spectral density after three hours in the supine position. When compared with the preceding period in the supine position low frequency power spectral density significantly increased in the sitting position at baseline and after three hours. One hour after drug administration, however, it did not significantly change.

\section{Metoprolol}

Compared with placebo, metoprolol significantly decreased the low frequency power spectral density after three hours in the sitting position. When compared with the preceding period in the supine position low frequency power spectral density significantly increased in the sitting position at baseline but not after one hour or three hours.

\section{Placebo}

When compared with the preceding period in the supine position, low frequency power spectral density significantly increased in sit- 
Table 4 Numerical results of low frequency power spectral densities of heart rate between $0.03 \mathrm{~Hz}$ and $0.15 \mathrm{~Hz}$. All values are normalised and expressed as percentages of total power spectral density

\begin{tabular}{|c|c|c|c|c|c|c|c|c|c|c|}
\hline \multirow[b]{2}{*}{ Phase } & \multicolumn{9}{|c|}{ Case no } & \multirow[b]{2}{*}{ Mean $(S D)$} \\
\hline & 1 & 2 & 3 & 4 & 5 & 6 & 7 & 8 & 9 & \\
\hline \multicolumn{11}{|c|}{ Gallopamil } \\
\hline Baseline: & & & & & & & & & & \\
\hline Supine & $43 \cdot 7$ & $23 \cdot 8$ & $40 \cdot 3$ & $73 \cdot 7$ & 3.5 & $13 \cdot 0$ & $32 \cdot 1$ & $27 \cdot 9$ & $18 \cdot 2$ & $30.69(20.55)$ \\
\hline Sitting & $23 \cdot 5$ & $20 \cdot 4$ & $49 \cdot 8$ & $18 \cdot 7$ & $14 \cdot 7$ & $42 \cdot 9$ & $72 \cdot 1$ & $25 \cdot 6$ & $4 \cdot 4$ & $30.23(20.92)$ \\
\hline \multicolumn{11}{|c|}{ After 1 hour: } \\
\hline Supine & $16 \cdot 5$ & 8.9 & $13 \cdot 2$ & $49 \cdot 0$ & $3 \cdot 2$ & 18.9 & $27 \cdot 3$ & $23 \cdot 8$ & $1 \cdot 7$ & $18.06(14.47)$ \\
\hline Sitting & $37 \cdot 6$ & $9 \cdot 8$ & 43.0 & $44 \cdot 3$ & $6 \cdot 2$ & 21.5 & 52.9 & $38 \cdot 4$ & $61 \cdot 6$ & $35.03(18.85)$ \\
\hline \multicolumn{11}{|c|}{ After 3 hours: } \\
\hline Supine & $80 \cdot 1$ & $32 \cdot 1$ & $14 \cdot 8$ & $44 \cdot 3$ & $44 \cdot 5$ & $26 \cdot 4$ & $13 \cdot 5$ & $14 \cdot 0$ & $4 \cdot 3$ & $30.44(23.35)$ \\
\hline Sitting & $11 \cdot 7$ & $12 \cdot 5$ & 38.9 & $38 \cdot 0$ & 34.0 & 39.9 & $30 \cdot 1$ & $26 \cdot 3$ & $37 \cdot 4$ & $29.87(10.99)$ \\
\hline \multicolumn{11}{|c|}{ Nifedipine } \\
\hline Baseline: & & & & & & & & & & \\
\hline Supine & $25 \cdot 0$ & $9 \cdot 9$ & $24 \cdot 2$ & $62 \cdot 8$ & 0.9 & $2 \cdot 4$ & $12 \cdot 9$ & 0.9 & $25 \cdot 1$ & $18 \cdot 23(19 \cdot 57)$ \\
\hline Sitting & $49 \cdot 2$ & $27 \cdot 2$ & $38 \cdot 7$ & $49 \cdot 2$ & 33.9 & $61 \cdot 7$ & $62 \cdot 9$ & $66 \cdot 2$ & $45 \cdot 0$ & $48.22(13.56)^{\star}$ \\
\hline \multicolumn{11}{|c|}{ After 1 hour: } \\
\hline Supine & $41 \cdot 2$ & $76 \cdot 7$ & $7 \cdot 1$ & 18.9 & $7 \cdot 1$ & $6 \cdot 7$ & $3 \cdot 6$ & $8 \cdot 1$ & $30 \cdot 5$ & $22 \cdot 21(24 \cdot 11)$ \\
\hline Sitting & $29 \cdot 6$ & $10 \cdot 8$ & $25 \cdot 3$ & $51 \cdot 0$ & $42 \cdot 4$ & $54 \cdot 5$ & $59 \cdot 7$ & $3 \cdot 2$ & $70 \cdot 8$ & $38.59(22.88)$ \\
\hline \multicolumn{11}{|c|}{ After 3 hours: } \\
\hline Supine & $9 \cdot 2$ & $9 \cdot 4$ & $39 \cdot 5$ & $5 \cdot 4$ & 13.5 & $37 \cdot 0$ & $1 \cdot 2$ & $6 \cdot 6$ & $3 \cdot 7$ & $13.94(14.24) \ddagger$ \\
\hline Sitting & $88 \cdot 3$ & $9 \cdot 8$ & $26 \cdot 4$ & $54 \cdot 6$ & $30 \cdot 7$ & $75 \cdot 6$ & $79 \cdot \overline{5}$ & $37 \cdot 2$ & $45 \cdot 0$ & $49.68(26.80) \dagger$ \\
\hline \multicolumn{11}{|c|}{ Metoprolol } \\
\hline Baseline: & & & & & & & & & & \\
\hline Supine & $62 \cdot 3$ & 9.9 & $30 \cdot 3$ & 13.9 & $9 \cdot 2$ & $29 \cdot 4$ & $11 \cdot 3$ & $10 \cdot 5$ & $21 \cdot 3$ & $22.01(17 \cdot 21)$ \\
\hline Sitting & $56 \cdot 4$ & $17 \cdot 7$ & $39 \cdot 3$ & $71 \cdot 4$ & $23 \cdot 0$ & $79 \cdot 3$ & $17 \cdot 5$ & $49 \cdot 4$ & $19 \cdot 4$ & $41.49(23.94)^{\star}$ \\
\hline \multicolumn{11}{|c|}{ After 1 hour: } \\
\hline Supine & $13 \cdot 5$ & $2 \cdot 9$ & $4 \cdot 1$ & 13.9 & $47 \cdot 5$ & $24 \cdot 1$ & $8 \cdot 6$ & $44 \cdot 0$ & $12 \cdot 9$ & $19.06(16.38)$ \\
\hline Sitting & $44 \cdot 6$ & 23.9 & 11.9 & $44 \cdot 7$ & $14 \cdot 1$ & $65 \cdot 6$ & $16 \cdot 5$ & $24 \cdot 7$ & $24 \cdot 3$ & $30.03(17.63)$ \\
\hline \multicolumn{11}{|c|}{ After 3 hours: } \\
\hline Supine & $18 \cdot 2$ & $48 \cdot 7$ & $14 \cdot 8$ & & $73 \cdot 5$ & $32 \cdot 2$ & $4 \cdot 8$ & $23 \cdot 0$ & $27 \cdot 9$ & $27 \cdot 47(22 \cdot 13)$ \\
\hline Sitting & $56 \cdot 9$ & $20 \cdot 8$ & $20 \cdot 4$ & $26 \cdot 6$ & $24 \cdot 4$ & 63.5 & $31 \cdot 7$ & $17 \cdot 5$ & $13 \cdot 7$ & $30.61(17.63)$ \\
\hline \multicolumn{11}{|c|}{ Placebo } \\
\hline Baseline: & & & & & & & & & & \\
\hline Supine & $12 \cdot 5$ & $1 \cdot 1$ & $37 \cdot 0$ & $35 \cdot 8$ & $28 \cdot 0$ & $36 \cdot 8$ & $42 \cdot 3$ & $25 \cdot 3$ & $12 \cdot 3$ & $25 \cdot 68(14 \cdot 11)$ \\
\hline Sitting & $38 \cdot 6$ & $54 \cdot 2$ & $27 \cdot 2$ & $55 \cdot 0$ & 56.0 & $78 \cdot 8$ & 43.5 & $23 \cdot 6$ & $37 \cdot 5$ & $46.04(17.00)^{\star}$ \\
\hline \multicolumn{11}{|c|}{ After 1 hour: } \\
\hline Supine & $32 \cdot 4$ & $2 \cdot 4$ & $33 \cdot 3$ & $41 \cdot 2$ & $11 \cdot 7$ & $6 \cdot 1$ & $7 \cdot 1$ & $13 \cdot 3$ & $1 \cdot 6$ & $16.57(14.98)$ \\
\hline Sitting & $21 \cdot 2$ & $7 \cdot 4$ & $13 \cdot 2$ & $38 \cdot 7$ & $29 \cdot 5$ & $57 \cdot 8$ & $47 \cdot 1$ & $34 \cdot 1$ & $25 \cdot 8$ & $30.53(15.98)$ \\
\hline \multicolumn{11}{|c|}{ After 3 hours: } \\
\hline Supine & 31.9 & $70 \cdot 1$ & $20 \cdot 8$ & $53 \cdot 6$ & $51 \cdot 1$ & $56 \cdot 2$ & $3 \cdot 8$ & $3 \cdot 7$ & $45 \cdot 5$ & $37 \cdot 41(23 \cdot 74)$ \\
\hline Sitting & $44 \cdot 4$ & $33 \cdot 4$ & $27 \cdot 0$ & $22 \cdot 8$ & $55 \cdot 1$ & 91.8 & $53 \cdot 4$ & 53.9 & $39 \cdot 1$ & $46.77(20.62)$ \\
\hline
\end{tabular}

ting body position at baseline but not after one hour or three hours.

\section{Discussion}

Compared with placebo gallopamil caused a mild but significant increase in heart rate after one hour in the supine position. The lack of an increase after the change to sitting and the fact that one subject developed an atrioventricular block II type Mobitz indicate that gallopamil inhibits sympathetic activation and prolongs atrioventricular conduction. Com-pared with placebo, heart rate after three hours was lower only in the sitting position but not in the supine position. This may be explained by the fact that under enhanced sympathetic tone the differences to placebo may become more obvious and finally reach significance.

Nifedipine caused reflex sympathetic activation with tachycardia, followed by an exponential decline in heart rate in all but one volunteer. The time constant of the decline had a remarkably low variance and was 34 (7) $\mathrm{min}$ in seven of the nine volunteers. One volunteer, however, showed a prolonged time constant of 83 minutes and may have had a prolonged reaction in his cardiovascular reflexes. Differences in drug disposition also could explain this phenomenon. Time domain heart rate variability with nifedipine significantly decreased after one hour indicating that parasympathetic activity was strongly overdriven by reflex sympathetic activity. With metoprolol time domain heart rate variability significantly increased showing a high parasympathetic activity after $\beta$ adrenergic blockade. In addition, there was a persistent negative chronotropic effect. The lowering effect of nifedipine on the rate-pressure product on exercise was caused by its lowering effect on maximal systolic blood pressure, though reflex tachycardia was no longer present after four hours. This is in good agreement with the finding, that reflex tachycardia declines exponentially with a time constant of $t_{d} \approx 34$ minutes, which means that after $3 t_{d} \approx$ 102 min there would be no further effect on heart rate.

Spectral analysis of $R R$ intervals and calculation of low frequency power spectral density as an index of sympathetic cardiac activity gave some interesting results. With gallopamil there were no differences between the supine and sitting positions. This probably means that the normal increase in low frequency power spectral density after the change in posture did not appear, because gallopamil inhibits cardiac sympathetic activation. In addition, compared with placebo, gallopamil significantly lowered low frequency power spectral density three hours after administration in the sitting position. Both findings sup- 
port the hypothesis that cardiac sympathetic activation is inhibited by gallopamil. This effect was seen in the spectral analysis of heart rate variability, though mean heart rate did not change within the subjects. This is not a contradiction because the spectral content of any signal can change without change of its mean value. In addition, it has been shown that time domain heart rate variability can change without change of mean heart rate during antiarrhythmic treatment. ${ }^{30}$ In summary, these findings can hardly be explained by a simple fixed delaying effect of the drugs on atrioventricular conduction because in this case the $R$ wave would be delayed only to the preceding atrial activation by a fixed lag time and the time series of the $R R$ intervals would be shifted in time but not changed in time or frequency domain characteristics. Hitherto it is not clear whether gallopamil exhibits a more complicated modulating effect on atrioventricular conduction, which may be related to sinus cycle duration and results in the observed decrease of low frequency power spectral density without significant increase in mean $R R$ interval duration. Furthermore, the site of action of gallopamil is not clear. As is known from animal experiments, it may act as an inhibitor of calcium dependent exocytotic noradrenaline and neuropeptide $\mathrm{Y}$ release $\mathrm{r}^{35-38}$ at the sites of signal transmission at the synaptic cleft or act directly as a modulating substance of the electromechanical coupling at the myocardium. ${ }^{38} 39$ To assess the sinus cycle duration related atrioventricular conductivity studies with continuous measurements of sinus rate and the atrioventricular conduction time should be undertaken.

With nifedipine the normal increase in low frequency power spectral density after the change to sitting was seen at baseline and after three hours but not after one hour. Time domain analysis showed that this can be explained by the reflex sympathetic activation which was still effective one hour after administration. Therefore the change to sitting body position did not add further measurable sympathetic activation.

With metoprolol there was the expected increase in low frequency power spectral density when posture changed to sitting at baseline. This was not observed after one hour and three hours, indicating that reflex sympathetic activation was blocked. Time domain heart rate variability accordingly increased showing increased parasympathetic activity.

In conclusion, gallopamil and metoprolol inhibit cardiac sympathetic activation. Whereas metoprolol also lowers heart rate which may be explained by a lower basal sympathetic tone, the inhibition of sympathetic activation by gallopamil may be related to a modulating effect on sinus rate and atrioventricular conduction but without significant decrease of heart rate. In contrast, nifedipine causes strong reflex sympathetic activation which lasts approximately 100 minutes.
This study was supported by the Sonderforschungsbereich 320 "Herzfunktion und ihre Regulation" within the Deutsche Forschungsgemeinschaft.

1 Akselrod S, Gordon D, Ubel FA, Shannon DC, Cohen RJ. Power spectrum analysis of heart rate fluctuation: quantitative probe of beat-to-beat cardiovascular control. Science 1981;213:220-2.

2 Akselrod S, Gordon D, Madwed J, Snidman N, Shannon DC, Cohen RJ. Hemodynamic regulation: investigation by spectral analysis. Am ₹ Physiol 1985;249:H867-75.

3 Cerutti S, Bianchi A, Baselli G, Civardi S, Guzzetti S, Malliani A, et al. Compressed spectral arrays for the analysis of $24-\mathrm{hr}$ heart rate variability signal: enhancement of parameters and data reduction. Comput Biomed Res 1988;22:424-41.

4 Lipsitz LA, Mietus J, Moody GB, Goldberger AJ. Spectra characteristics of heart rate variability before and during postural tilt. Relations to age and risk of syncope. Circulation 1990;81:1803-10.

5 Lombardi F, Sandrone G, Pernpruner S, Sala R, Garimoldi $M$, Cerutti $S$, et al. Heart rate variability as an index of sympathovagal interaction after acute myocardial infarction. Am $\mathcal{F}$ Cardiol 1987;60:1239-45.

6 Pagani M, Lombardi F, Guzzetti S, Rimoldi O, Furlan R, Pizzinelli P, et al. Power spectral analysis of heart rate and arterial pressure variabilities as a marker of sympatho-vagal interaction in man and in conscious dog. Circ Res 1986;59:178-93.

7 Pomeranz B, Macaulay R, Caudill M. Assessment of autonomic function in humans by heart rate spectral analysis. Am f Physiol 1985;248: H151-3.

8 Saul J, Rea R, Eckberg D, Berger R, Cohen R. Heart rate and muscle sympathetic nerve variability during reflex changes of autonomic activity. Am $\mathcal{F}$ Physiol 1990;258: H713-21.

9 Taylor M. Use of random excitation and spectral analysis in the study of frequency-dependent parameters of the cardiovascular system. Circ Res 1966;18:585-95.

10 Baselli G, Cerutti S, Civardi S, Liberati D, Lombardi F, Malliani A, et al. Spectral and cross-spectral analysis of heart rate and arterial blood pressure variability signals. Comput Biomed Res 1986;19:520-34.

11 De Boer RW, Karemaker JM, Strackee J. Comparing spectra of a series of point events particularly for heart rate variability data IEEE Trans Biomed Eng 1984, BME-31:384-7.

12 De Boer RW, Karemaker JM, Strackee J. Haemodynamic fluctuations and baroreflex sensitivity in humans: a beatfluctuations and baroreflex sensitivity in human

13 Furlan R, Guzzetti S, Crivellaro W, Dassi S, Tinelli M, Baselli $G$, et al. Continuous 24-hour assessment of the neural regulation of systemic arterial pressure and $R R$ variabilities in ambulant subjects. Circulation 1990;81 $537-47$

14 Myers G, Martin G, Magid N. Power spectral analysis of heart rate variability in sudden cardiac death: comparison to other methods. IEEE Trans Biomed Eng 1986;BME-33:1149-56.

15 Bellavere F, Balzani I, De Masi G, Carraro M, Carenza P, Cobelli C, et al. Power spectral analysis of heart-rate variations improves assessment of diabetic cardiac autonomic neuropathy. Diabetes $1992 ; 41: 633-40$

16 Bianchi A, Bontempi B, Cerutti S, Gianoglio P, Comi G Sora MG. Spectral analysis of heart rate variability sig-
Sorat nal and respiration in diabetic subjects. Med Biol Eng Comput 1990;28:205-11.

17 Casolo G, Balli E, Taddei T, Amuhasi J, Gori C. Decreased spontaneous heart rate variability in congestive heart failure. Am F Cardiol 1989;64:1162-7.

18 Cripps TR, Malik M, Farrell TG, Camm AJ. Prognostic value of reduced heart rate variability after myocardial infarction: clinical evaluation of a new analysis method. Br Heart $\mathcal{f} 1991 ; 65: 14-9$.

19 Martin GJ, Magid NM, Myers G. Heart rate variability and sudden death secondary to coronary artery disease during ambulatory electrocardiographic monitoring. $\mathrm{Am}$ during ambulatory elect

20 Rich M, Saini J, Kleiger R, Carney R, teVelde A, Freedland $\mathrm{K}$. Correlation of heart rate variability with clinical and angiographic variables and late mortality after coronary angiography. Am $f$ Cardiol 1988;62: 714-7

21 Ewing DJ, Neilson JMM, Travis P. New method for assessing cardiac parasympathetic activity using 24 hou electrocardiograms. Br Heart $\mathcal{F}$ 1984;52:396-402.

22 La Rovere MT, Specchia G, Mortara A, Schwartz PJ Baroreflex sensitivity, clinical correlates, and cardiovascular mortality among patients with a first myocardial cular mortality among patients with a

23 Schwartz PJ, Vanoli E, Stramba-Badiali M, De Ferrari GM, Billman GE, Foreman RD. Autonomic mechanisms and sudden death. New insights from analysis of baroreceptor reflexes in conscious dogs with and of baroreceptor reflexes in conscious dogs with and without

24 Bigger JT Jr, Kleiger RE, Fleiss JL, Rolinitzky LM, Steinman RC, Miller JP, and the Multicenter PostInfarction Research Group. Components of heart rate variability measured during healing of acute myocardial infarction. Am 7 Cardiol 1988;61:208-15. 
25 Ewing DJ. Heart rate variability: an important new risk factor in patients following myocardial infarction. $A m \mathcal{F}$ Cardiol 1991;14:683-5.

26 Kleiger RE, Philip Miller J, Thomas Bigger J, Moss AJ, and the Multicenter Post-Infarction Research Group. Decreased heart rate variability and its association with increased mortality after acute myocardial infarction. Am $\mathcal{F}$ Cardiol 1987;59:256-62.

27 Malik M, Farrell T, Cripps, T, Camm AJ. Heart rate variability in relation to prognosis after myocardial infarction: selection of optimal processing techniques. Eur Heart f 1989;10: 1060-74.

28 Malik M, Camm AJ. Significance of long term components of heart rate variability for the further prognosis after acute myocardial infarction. Cardiovasc Res 1990;24:793-803.

29 Bigger JT Jr, Albrecht P, Steinmann RC, Rolnitzky LM, Cohen RI. Comparison of time and frequency domainbased measures of cardiac parasympathetic activity in based measures of cardiac parasympathetic activity in Cardiol 1989;64:536-8.

30 Zuanetti G, Latinin R, Neilson JMM, Schwartz PJ, Ewing DJ, and the Antiarrhythmic Drug Evaluation Group (ADEG). Heart rate variability in patients with ventricular arrhythmias: effect of antiarrhythmic drugs. $\mathcal{F} \mathrm{Am}$ Coll Cardiol 1991;17:604-12.

31 The Cardiac Arrhythmia Suppression Trial (CAST) Investigators. Preliminary report: effect of encainide and flecainide on mortality in a randomized trial of arrhythmia suppression after myocardial infarction. $N$ Engl $f$ Med 1989;21:406-12.

32 Bekheit S, Tangella M, El-Sakr A, Rasheed O, Craelius $W$, EL-Sherif N. Use of heart rate spectral analysis to study the effects of calcium channel blockers on sympa- thetic activity after myocardial infarction. Am Heart $\mathcal{F}$ 1990;119:79-85.

33 Wesseling $\mathrm{KH}$. Finapres-Kontinuierliche, nichtinvasive arterielle Blutdruckmessung am Finger nach der Methode von Penaz. In: Meyer-Sabellek W, Gotzen R, Methode von Penaz. In: Meyer-Sabellek W, Gotzen R, Steinkopff, 1988:45-59.

34 Rompelman O, Snijders J, van Spronsen C. The measurement of heart rate variability spectra with the help of a personal computer. IEEE Trans Biomed Eng 1982; BME-29:503-10

35 Schömig A, Haass M, Richardt G. Catecholamine release and arrhythmias in acute myocardial ischaemia. Eur Heart $\mathcal{f} 1991 ; 12$ (suppl F):38-47.

36 Schömig A, Haass $M$. Neuropeptide $Y$-a marker for noradrenaline exocytosis. Ann NY Acad Sci 1990; 611:447-9.

37 Haass M, Förster C, Kranzhöfer R, Richardt G, Schömig A. Role of calcium channels and protein kinase $C$ for release of norepinephrine and neuropeptide Y. $\mathrm{Am} f$ release of norepinephrine

38 Fleckenstein A Tritthart $H$, Fleckenstein B, Herbst A, Grün G. A new group of competitive $\mathrm{Ca}$ antagonists (iproveratril, D 600 , prenylamine) with highly potent nhibitory effects on excitation-contraction coupling in mammalian myocardium. Pflugers Arch 1969;307: R25.

39 Fleckenstein A, Fleckenstein B, Späh F, Byon YK. Gallopamil (D 600)-calcium antagonist of high potency and specificity. Effects on the myocardium and pacemakers. In: Kaltenbach $M$, Hopf $\mathrm{R}$, eds. Gallopamil: pharmacological and clinical profile of a calcium antagonist. Berlin: Springer, 1984:1-28. 